ARGONNE NATIONAL LABORATORY

P. O. Box 5207

Chicago 80 , Illinois

\title{
TEMPERATURE AND STRESS DISTRIBUTION IN \\ SPHERES, RODS, TUBES, AND PLATES \\ IN WHICH THE HEAT SOURCE IS WITHIN \\ THE BOUNDARIES OF THE SOLIDS
}

by

J.C. Carter

Reactor Engineering \& Services Division

\section{September 7, 1951}

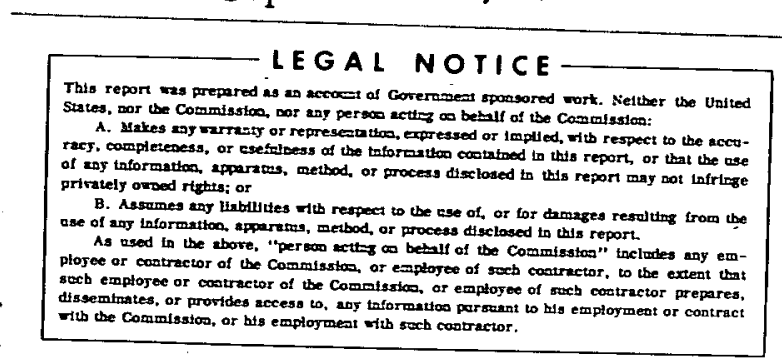

Operated by The University of Chicago under

Contract W-31-109-eng-38

This report has been photostated to fill your request as our supply of copies was exhausted. If you should find that you do not need to retain this copy permanently in your files, we would greatly appreciate your returning it to TIS so that it may be used to fill future requests from other AEC installations. 


\section{DISCLAIMER}

This report was prepared as an account of work sponsored by an agency of the United States Government. Neither the United States Government nor any agency Thereof, nor any of their employees, makes any warranty, express or implied, or assumes any legal liability or responsibility for the accuracy, completeness, or usefulness of any information, apparatus, product, or process disclosed, or represents that its use would not infringe privately owned rights. Reference herein to any specific commercial product, process, or service by trade name, trademark, manufacturer, or otherwise does not necessarily constitute or imply its endorsement, recommendation, or favoring by the United States Government or any agency thereof. The views and opinions of authors expressed herein do not necessarily state or reflect those of the United States Government or any agency thereof. 


\section{DISCLAIMER}

Portions of this document may be illegible in electronic image products. Images are produced from the best available original document. 


\section{INTRODUCTION}

Energy may exist as heat in solids by virtue of their resistance to the flow of electric current, by induction or by nuclear reaction. The problem of determining the temperature distribution and the thermal stress occasioned by temperature gradients is especially important in the design of equipment in which thermal energy is transferred at high rates. The ratio of the cross section of the solids to the heat transfer surfaces is critical since the heat is transferred by conduction to the surface of the solid and then by forced convection from the surface to the coolant. If the path for heat flow is too long or the rate of increase of energy level is too fast the temperature gradient through the solid can be great enough to create excessive thermal stress or disintegration.

The most common geometrical configurations considered are spheres, rods, round tubes and plates. These will be treated with the simplifying assumptions that the heat generation is uniform per unit volume of material and that the thermal conductivity, coefficient of expansion, and modulus of elasticity remain constant.

The equations for temperature distribution are developed. These are substituted into the stress equations (1) and curves using dimensionless groups in the stress equations as coordinates are presented.

It is impossible to anticipate all the mathematical expressions for the source function or the boundary conditions, therefore the form of the general solution of the cases most frequently encountered is given and it is hoped the technique employed will aid in the solution of specific cases.

SYMBOLS
$a=$ radius of inner surface
$\mathrm{b}=$ radius of outer surface
$\mathrm{D}=$ flexural rigidity
$E=$ modulus of elasticity
$\mathrm{H}=$ heat per unit area
$h=$ heat transfer coefficient
$\mathbf{k}=$ thermal conductivity
$L=$ width
$M=$ bending moment
$q=$ heat per. unit volume
$\mathbf{r}=$ radius
$S=$ surface

$T=$ temperature
$\theta=\mathrm{T}-\mathrm{T}_{\mathbf{s}}$
$\sigma=$ stress
$\nu=$ Poisson's ratio
$\alpha=$ coefficient of thermal ex- pansion

Subscripts:

$c=$ coolant

s = surface

$t=$ tangential

S $=$ surface

$\mathbf{z}=$ longitudinal

(1) Theory of Elasticity - Timoshenko 


\section{SPHERES}

Energy in the form of heat per unit volume is generated uniformly and steadily within the metal of the sphere and transferred by conduction to the surface from whence it is transferred by forced convection to the coolant.

The heat energy stored in unit time in a spherical shell is determined in the following manner. The rate of heat flow $\mathrm{H}_{\mathrm{r}}$ at any distance $r$ is equal to $\left(-4 \pi r^{2} \mathrm{~K} \partial \mathrm{T} / \partial \mathrm{r}\right)$, and

$$
\mathrm{H}_{2}=\mathrm{H}_{1}-\left(\frac{\partial \mathrm{H}}{\partial \mathrm{r}}\right) \mathrm{dr}
$$

When no heat source exists within the sphere the thermal energy stored in unit time in a spherical element $\mathrm{dr}$ is

$$
\left(\frac{\partial \mathrm{H}_{\mathrm{r}}}{\partial \mathrm{r}}\right) \mathrm{dr}=\left[-4 \pi \mathrm{K}\left(2 \mathrm{r} \frac{\partial \mathrm{T}}{\partial \mathrm{r}}+\mathrm{r}^{2} \frac{\partial^{2} \mathrm{~T}}{\partial \mathrm{r}^{2}}\right)\right] \mathrm{dr}
$$

With heat generation, the heat in the element $d r$ is ( $q 4 \pi r^{2} d r$ )

Equating 1 and 2

$$
\frac{\partial^{2} \mathrm{~T}}{\partial \mathrm{r}^{2}}+\frac{2}{\mathrm{r}}\left(\frac{\partial \mathrm{T}}{\partial \mathrm{r}}\right)+\frac{\mathrm{q}}{\mathrm{K}}=0
$$

The general solution of this equation for the steady state is

$$
\begin{array}{ll}
\theta=\frac{q r^{2}}{6 K}-\frac{C_{1}}{r}+C_{2} & \text { where } \theta=T-T_{s} \\
\theta=\frac{q}{6 K}\left(b^{2}-r^{2}\right) & \text { for solid sphere } \\
\theta=\frac{q}{3 K}\left[\left(\frac{b^{2}-r^{2}}{2}\right)+a^{3}\left(\frac{1}{b}-\frac{1}{r}\right)\right] & \text { for hollow sphere }
\end{array}
$$

The equations for stress distribution in a solid sphere are

$$
\begin{aligned}
& \sigma_{r}=\frac{2 \alpha E}{1-\nu}\left(\frac{1}{b^{3}} \int_{0}^{b} T r^{2} d r-\frac{1}{r^{3}} \int_{0}^{r} T r^{2} d r\right) \\
& \sigma_{t}=\frac{\alpha E}{1-\nu}\left(\frac{2}{b^{3}} \int_{0}^{b} T r^{2} d r+\frac{1}{r^{3}} \int_{0}^{r} T r^{2} d r-T\right)
\end{aligned}
$$


Substituting the value of $\theta=T$ for the solid sphere into the equations for stress

$$
\left.\begin{array}{l}
\sigma_{r}=\frac{\alpha E q}{(1-\nu) 15 K}\left(r^{2}-b^{2}\right) \cdot \frac{r^{2}}{b^{2}}-1=\frac{15 K \sigma_{r}(1-\nu)}{\alpha E q b^{2}} \\
\sigma_{t}=\frac{\alpha E q}{(1-\nu) 15 K}\left(2 r^{2}-b^{2}\right) \quad \frac{2 r^{2}}{b^{2}}-1=\frac{15 K \sigma_{T}(1-\nu)}{\alpha E q b^{2}}
\end{array}\right\} \begin{gathered}
\text { Fig. } \\
I
\end{gathered}
$$

The equations for stress distribution in a hollow sphere are

$$
\begin{aligned}
& \sigma_{r}=\frac{2 \alpha E}{1-\nu}\left\{\frac{r^{3}-a^{3}}{\left(b^{3}-a^{3}\right) r^{3}} \int_{a}^{b} \operatorname{Tr}^{2} d r-\frac{1}{r^{3}} \int_{a}^{r} T r^{2} d r\right\} \\
& \sigma_{t}=\frac{2 \alpha E}{1-\nu}\left\{\frac{2 r^{3}+a^{3}}{2\left(b^{2}-a^{3}\right) r^{3}} \int_{a}^{b} T r^{2} d r+\frac{1}{2 r^{3}} \int_{a}^{r} T r^{2} d r-\frac{1}{2} T\right\}
\end{aligned}
$$

Substituting the value of $\theta=\mathrm{T}$ for the hollow sphere into the equations for stress

$$
\begin{aligned}
\sigma_{r}= & \frac{2 \alpha E q}{3 K(1-\nu)}\left\{\frac{r^{3}-a^{3}}{\left(b^{3}-a^{3}\right) r^{3}}\left[\frac{b^{5}}{15}-\frac{a^{3} b^{2}}{3}+\frac{3 a^{5}}{5}-\frac{a^{6}}{3 b}\right]\right. \\
& \left.\frac{1}{r^{3}}\left[\frac{r^{3} b^{2}}{6}-\frac{r^{5}}{10}+\frac{a^{3} r^{3}}{3 b}-\frac{a^{3} r^{2}}{2}-\frac{a^{3} b^{2}}{6}+\frac{3 a^{5}}{5}-\frac{a^{6}}{3 b}\right]\right\} \\
\sigma_{t}= & \frac{2 \alpha E q}{3 K(1-\nu)}\left\{\frac{2 r^{3}+a^{3}}{2\left(b^{3}-a^{3}\right) r^{3}}\left[\frac{b^{5}}{15}-\frac{a^{3} b^{2}}{3}+\frac{3 a^{5}}{5}-\frac{a^{6}}{3 b}\right]\right. \\
& +\frac{1}{2 r^{3}}\left[\frac{r^{3} b^{2}}{6}-\frac{r^{5}}{10}+\frac{a^{3} r^{3}}{3 b}-\frac{a^{3} r^{2}}{2}-\frac{a^{3} b^{2}}{6}-\frac{3 a^{5}}{5}-\frac{a^{6}}{3 b}\right] \\
& \left.-\frac{1}{2}\left[\left(\frac{b^{2}-r^{2}}{2}\right)+a^{3}\left(\frac{1}{r}-\frac{1}{b}\right)\right]\right\}
\end{aligned}
$$

Equations 13 and 14 are shown on Figs. 2 and 3. 

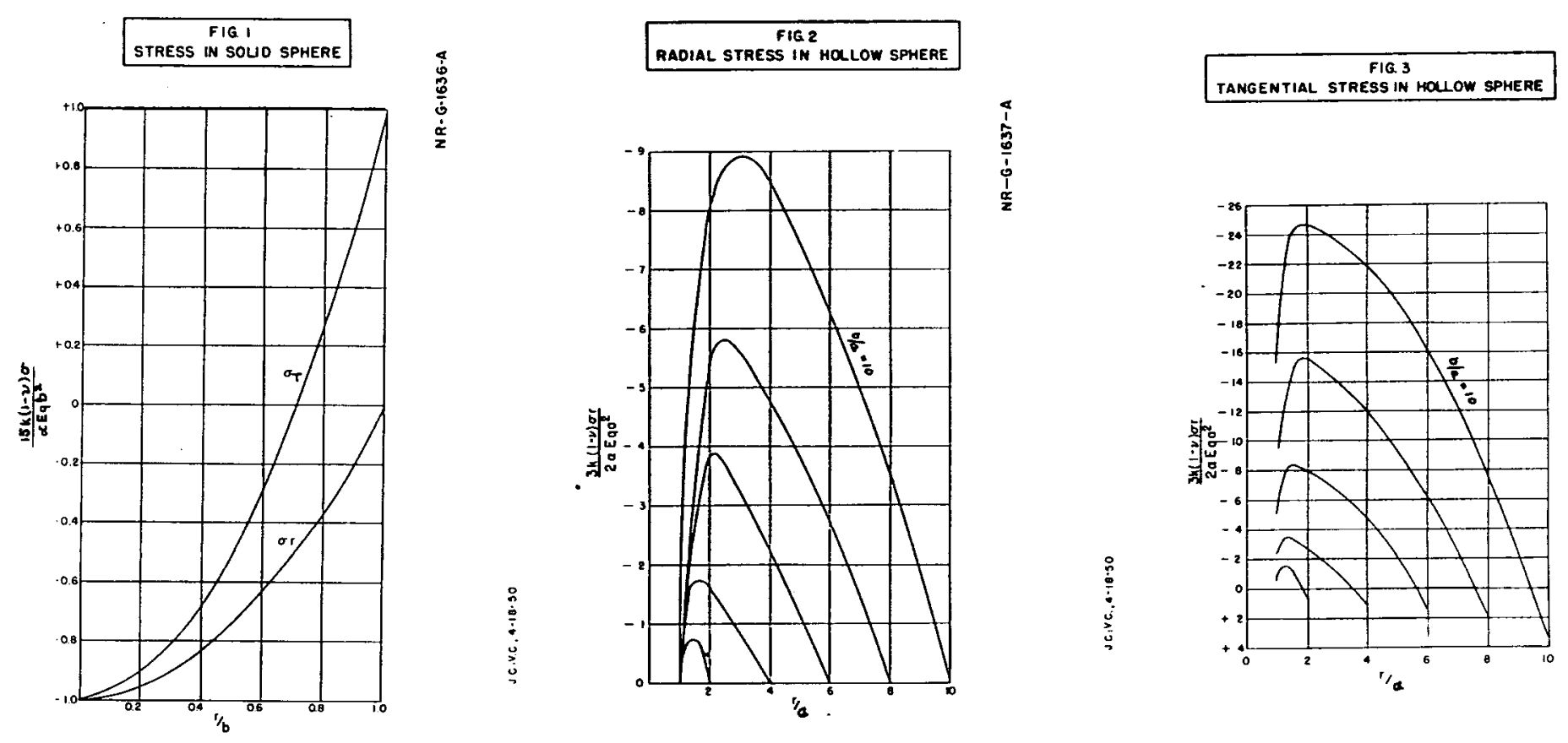

$\underline{\text { RODS }}$

The equation corresponding to equation (3) for spheres is

$$
\frac{\partial^{2} T}{\partial r^{2}}+\frac{1}{r} \frac{\partial T}{\partial r}-\frac{q}{K}=0
$$

The general solution of this equation for the steady state is

$$
\begin{aligned}
& \theta=-\frac{q r^{2}}{4 K}+C_{1} \ln r+C_{2} \quad \theta=T-T_{s} \\
& \theta=\frac{q}{4 K}\left(b^{2}-r^{2}\right)
\end{aligned}
$$

The stress equations for long solid cylinders are

$$
\begin{aligned}
& \sigma_{r}=\frac{\alpha E}{1-\nu}\left(\frac{1}{b^{2}} \cdot \int_{0}^{b} \operatorname{Trdr}-\frac{1}{r^{2}} \int_{0}^{r} \operatorname{Trdr}\right) \\
& \sigma_{t}=\frac{\alpha E}{1-\nu}\left(-T+\frac{1}{b^{2}} \int_{0}^{b} T r d r+\frac{1}{r^{2}} \int_{0}^{r} T r d r\right) \\
& \sigma_{z}=\frac{\alpha E}{1-\nu}\left(-T+\frac{2}{b^{2}} \int_{0}^{b} T r d r\right)
\end{aligned}
$$


Substituting the temperature function $\theta=T$ in equations 18,19 and 20

$$
\begin{aligned}
& \sigma_{r}=\frac{E \alpha q}{16 K(1-\nu)}\left(r^{2}-b^{2}\right) \\
& \sigma_{t}=\frac{E \alpha q}{16 K(1-\nu)}\left(3 r^{2}-b^{2}\right) \\
& \sigma_{z}=\frac{E \alpha q}{16 K(1-\nu)}\left(4 r^{2}-2 b^{2}\right)
\end{aligned}
$$

Fig. 4

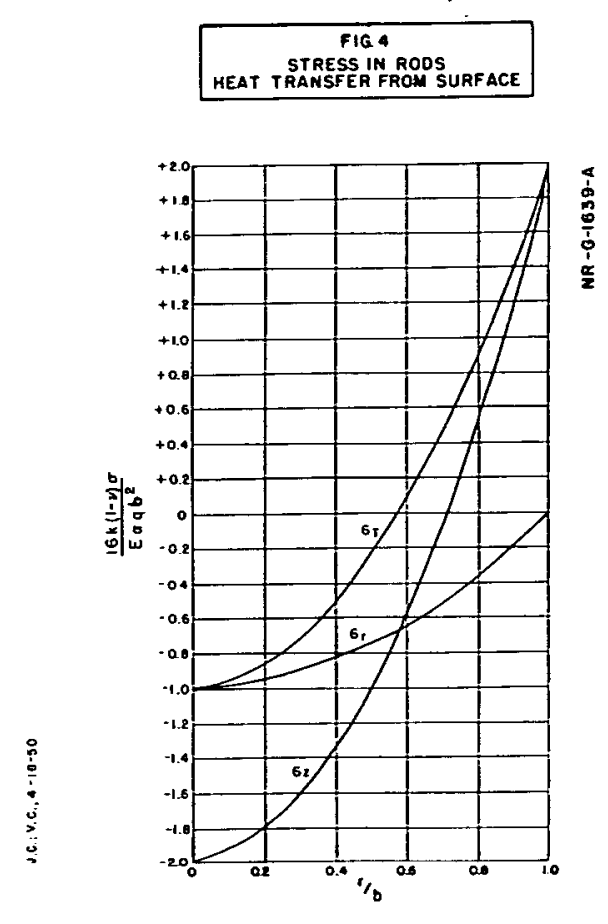




\section{$\underline{\text { TUBES }}$}

The general equation for temperature distribution is the same as that for rods but there are more combinations of boundary conditions. Heat may be transferred from both the outside and inside surface and at different rates.

The general equations of stress for long hollow cylinders are

$$
\begin{aligned}
& \sigma_{\dot{r}}=\frac{E \alpha}{(1-\nu) r^{2}}\left[\frac{r^{2}-a^{2}}{b^{2}-a^{2}} \int_{a}^{b} \operatorname{Trdr}-\int_{a}^{r} \operatorname{Trdr}\right] \\
& \sigma_{t}=\frac{E \alpha}{(1-\nu) r^{2}}\left[\frac{r^{2}+a^{2}}{b^{2}-a^{2}} \int_{a}^{b} \operatorname{Trdr}-\int_{a}^{r} \operatorname{Trdr}-\operatorname{Tr}^{2}\right] \\
& \sigma_{z}=\frac{E \alpha}{1-\nu}\left[T-\frac{2}{b^{2}-a^{2}} \int_{a}^{b} \operatorname{Trdr}\right]
\end{aligned}
$$

Case I Heat transfer from both sides but coolant temperature not equal on inside and outside surfaces of tube

$$
\begin{aligned}
& \theta=-\frac{q r^{2}}{4 K}+C_{1} \ln r+C_{2} \\
& \text { Let } \theta \text { or } u=T-T_{c} \quad \gamma=\frac{q}{2 K} \quad \phi=\frac{h_{c}}{K}
\end{aligned}
$$

$$
\frac{\mathrm{du}}{\mathrm{dr}}=-\gamma \mathrm{r}+\frac{\mathrm{C}_{1}}{\mathrm{r}}
$$

$u=-\frac{\gamma r^{2}}{2}+C_{1} \ln r+C_{2}$

$$
\begin{array}{lll}
\text { at } r=a & \frac{\overline{d u}}{d r}-\phi u_{a}=0 & C_{1}=\left(\phi u_{a}+\gamma a\right) a \\
\text { at } r=b & \frac{d u}{d r}+\phi u_{b}=0 & C_{1}=\left(-\phi u_{b}+\gamma b\right) b \\
\text { at } r=a & u_{a}=-\frac{\gamma}{2} a^{2}+C_{1} \ln a+C_{2} & C_{2}=\left(\frac{C_{1}}{a}-\gamma a\right) \frac{1}{\phi}+\frac{\gamma}{2} a^{2}-C_{1} \ln a \\
\text { at } r=b \quad u_{b}=\left(-\frac{C_{1}}{b}+\gamma b\right) \frac{1}{\phi} & C_{2}=\frac{1}{\phi}\left(-\frac{C_{1}}{b}+\gamma b\right)+\frac{\gamma}{2} b^{2}-C_{1} \ln b \\
C_{1}=\frac{\gamma\left(\frac{a}{\phi}+\frac{b}{\phi}-\frac{a^{2}}{2}+\frac{b^{2}}{2}\right)}{\left(\frac{1}{\phi a}+\frac{1}{\phi b}-\ln a+\ln b\right)} &
\end{array}
$$

The procedure in this case would be to determine (u) for specific conditions and then substitute in stress equations. 
Case II Heat removed from inner surface of tube only.

$$
\begin{array}{lcc}
\frac{\mathrm{d} \theta}{\mathrm{d} r} & \text { at } \mathrm{r}=\mathrm{b} & \mathrm{c}_{1}=\frac{\mathrm{q}}{2 \mathrm{~Kb}^{2}} \\
\text { at } \mathrm{r}=\mathrm{a} & \theta=0 & \mathrm{C}_{2}=\frac{\mathrm{qa}}{4 \mathrm{~K}}-\frac{\mathrm{q}}{2 \mathrm{~K}} \mathrm{~b}^{2} \ln \mathrm{a} \\
\theta=\frac{\mathrm{q}}{2 \mathrm{~K}} & \left\{\mathrm{~b}^{2} \ln \frac{\mathrm{r}}{\mathrm{a}}-\left(\frac{\mathrm{r}^{2}-\mathrm{a}^{2}}{2}\right)\right\}
\end{array}
$$

Substituting $\theta$ into general equations of stress

$$
\begin{aligned}
\sigma_{r} & =\frac{E \alpha q}{16 K(1-\nu) r^{2}} \quad\left[\frac{r^{2}-a^{2}}{b^{2}-a^{2}}\left(4 b^{4} \ln \frac{b}{a}-3 b^{4}+4 a^{2} b^{2}-a^{4}\right)\right. \\
& \left.\left.+2 b^{2} r^{2}\left(1-2 \ln \frac{r}{a}\right)-2 a^{2}\left(b^{2}+r^{2}\right)+r^{4}+a^{4}\right)\right] \\
\sigma_{t} & =\frac{E \alpha q}{16 K(1-\nu) r^{2}}\left[\frac{r^{2}+a^{2}}{b^{2}-a^{2}}\left(4 b^{4} \ln \frac{b}{a}-3 b^{4}+4 a^{2} b^{2}-a^{4}\right)\right. \\
& \left.-2 b^{2} r^{2}\left(1+2 \ln \frac{r}{a}\right)+2 a^{2}\left(b^{2}-r^{2}\right)+3 r^{4}-a^{4}\right] \\
\sigma_{z} & =\frac{-E \alpha q}{16 K(1-\nu)}\left[8 b^{2} \ln \frac{r}{a}-4 r^{2}+4 a^{2}-\frac{2}{b^{2}-a^{2}}\left(4 b^{4} \ln \frac{b}{a}-3 b^{4}\right.\right. \\
& \left.\left.+4 a^{2} b^{2}-a^{4}\right)\right]
\end{aligned}
$$

Equations 26, 27 and 28 are shown on Figs. 5, 6 and 7 .
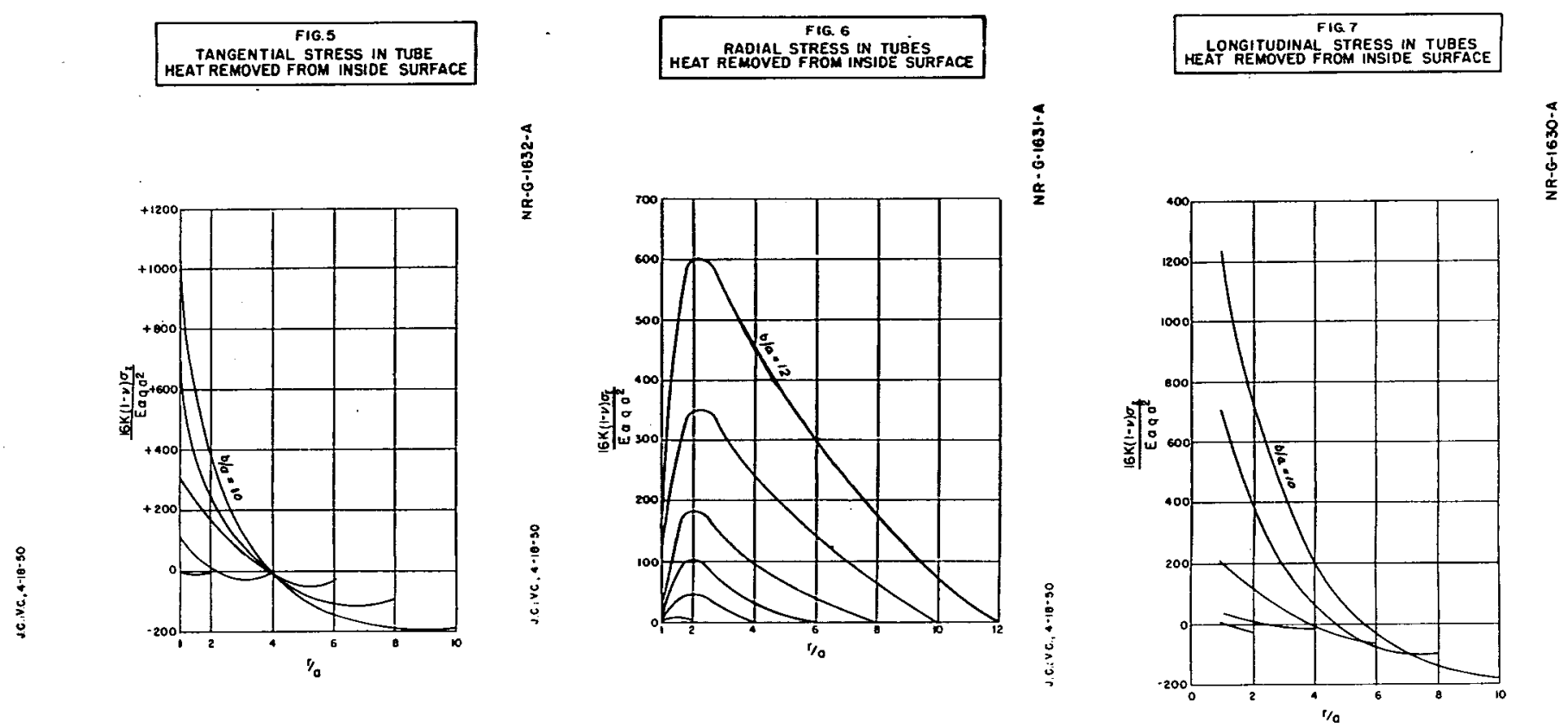
Case III Heat removed from the outer surface of tube only.

$$
\begin{array}{lcc}
\text { at } r=a & \frac{d \theta}{d r}=0 & C_{1}=\frac{q}{2 \mathrm{Ka}^{2}} \\
\text { at } r=b & \theta=0 & C_{2}=\frac{q b^{2}}{4 K}-\frac{q a^{2}}{2 K \ln b} \\
\theta=\frac{q}{2 K} & \left(\frac{b^{2}-r^{2}}{2}-a^{2} \ln \frac{b}{r}\right)
\end{array}
$$

Substituting $\theta-\mathrm{T}$ into general equations of stress

$$
\begin{aligned}
\sigma_{r} & =\frac{E \alpha q}{16 K(1-\nu) r^{2}}\left[\frac{r^{2}-a^{2}}{b^{2}-a^{2}}\left\{b^{4}-4 a^{2} b^{2}+3 a^{4}+4 a^{4} \ln \frac{b}{a}\right\}\right. \\
& \left.-\left\{2 r^{2} b^{2}-r^{4}-4 a^{2} r^{2} \ln \frac{b}{r}-2 a^{2} r^{2}-2 a^{2} b^{2}+3 a^{4}+8 a^{4} \ln \frac{b}{a}\right\}\right] \\
\sigma_{t} & =\frac{E a q}{16 K(1-\nu) r^{2}}\left[\frac{r^{2}+a^{2}}{b^{2}-a^{2}}\left\{b^{4}-4 a^{2} b^{2}+3 a^{4}+4 a^{4} \ln \frac{b}{a}\right\}\right. \\
& -\left\{2 r^{2} b^{2}-r^{4}-4 a^{2} r^{2} \ln \frac{b}{r}-2 a^{2} r^{2}-2 a^{2} b^{2}+3 a^{4}+4 a^{4} \ln \frac{b}{a}\right\} \\
& \left.-r^{2}\left\{4 b^{2}-4 r^{2}-8 a^{2} \ln \frac{b}{r}\right\}\right] \\
\sigma_{z} & =\frac{-E \alpha q}{16 K(1-\nu)}\left[4 b^{2}-4 r^{2}-8 a^{2} \ln \frac{b}{r}-\frac{2}{b^{2}-a^{2}}\right. \\
& \left.\left\{b^{4}-4 a^{2} b^{2}+3 a^{4}+4 a^{4} \ln \frac{b}{a}\right\}\right]
\end{aligned}
$$

Equations 30,31 and 32 are shown on Figs. 8, 9 and 10 

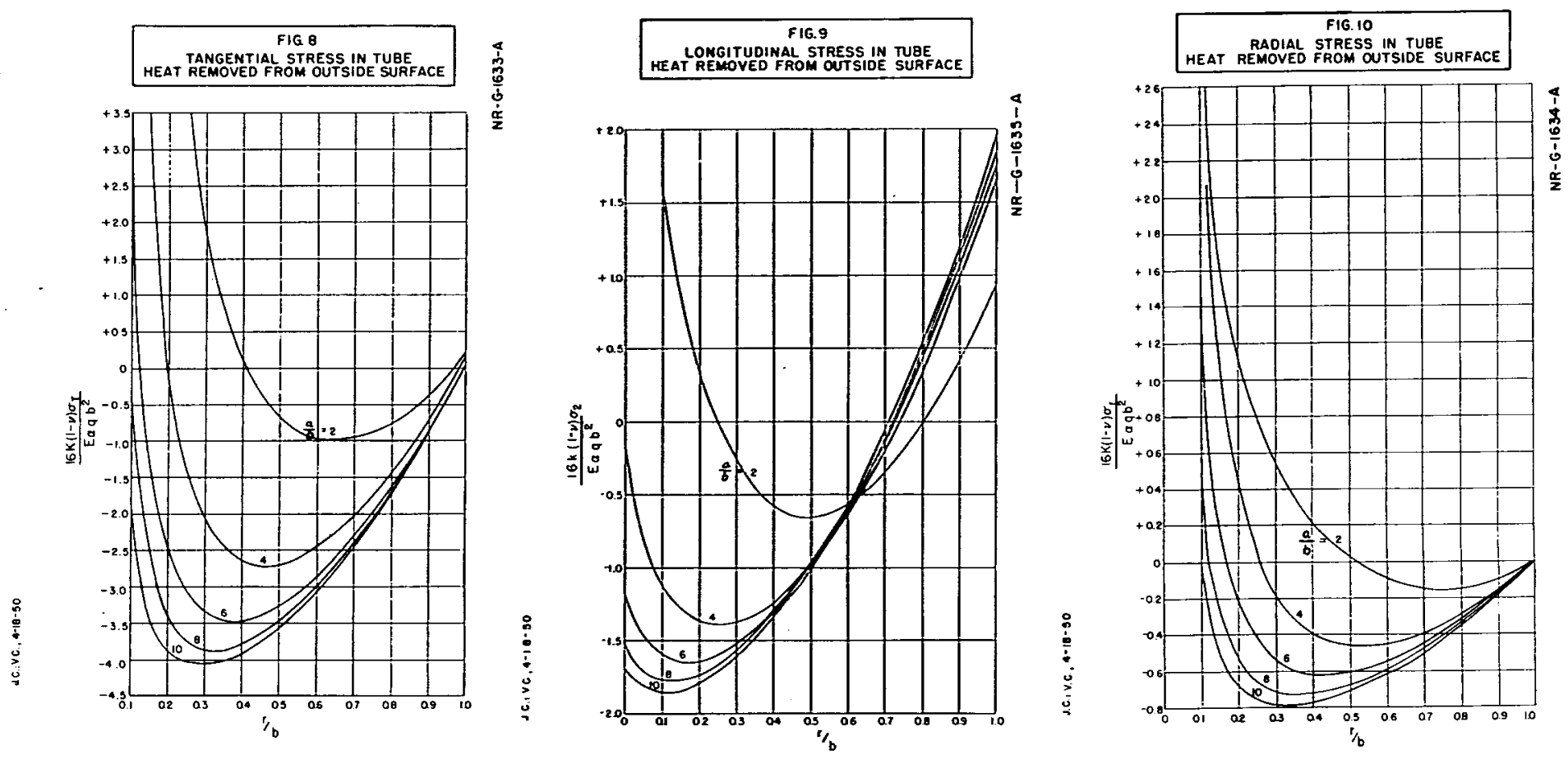

\section{PLATES}

The rate of heat flow at any distance $(x)$ in a plate $L$ units thick, is $H_{x}=-K S \frac{\partial T}{\partial x}$

$$
\mathrm{H}_{2}=\mathrm{H}_{1}-\frac{\partial \mathrm{H}_{\mathbf{x}}}{\partial \mathrm{x}} \mathrm{dx}
$$

With no heat source $\frac{\partial H_{x}}{\partial x} d x=-K S \frac{\partial^{2} T}{\partial x^{2}} d x$

If heat is produced in the plate at the rate (qSdx)

$$
\begin{aligned}
& \mathrm{KS} \frac{\partial^{2} T}{\partial \mathrm{x}^{2}} \mathrm{dx}+\mathrm{qSdx}=0 \\
& \theta=-\frac{\mathrm{qx^{2 }}}{2 \mathrm{~K}}+\mathrm{C}_{1} \mathrm{x}+\mathrm{C}_{2} \quad \cdot \theta=\mathrm{T}-\mathrm{T}_{\mathrm{S}}
\end{aligned}
$$

Case I Heat generation constant and coolant temperature equal on both sides

$$
\begin{array}{lrl}
\text { at } \mathrm{x}=\frac{\mathrm{L}}{2} & \frac{\mathrm{d} \theta}{\mathrm{dx}}=0 & \mathrm{C}_{1}=\frac{\mathrm{qL}}{2 \mathrm{~K}} \\
\text { at } \mathrm{x}=0 \text { or } \mathrm{L} \quad \theta=0 & \mathrm{C}_{2}=0 \\
\theta=-\frac{\mathrm{q} \mathrm{x}^{2}}{2 \mathrm{~K}}+\frac{\mathrm{qLx}}{2 \mathrm{~K}} &
\end{array}
$$


Case II Heat generation constant and coolant temperature not equal on both sides

$$
\begin{aligned}
& K \frac{d^{2} T}{d x^{2}}+q=0 \\
& \text { Let } \mathrm{u}=\mathrm{T}-\mathrm{T}_{\mathrm{c}} \quad \gamma=\frac{\mathrm{q}}{\mathrm{K}} \\
& \theta=\frac{\mathbf{h}_{\mathbf{c}}}{\mathrm{K}} \\
& \frac{d^{2} u}{d x^{2}}+\gamma=0 \\
& \frac{d u}{d x}=-\gamma x+C_{1} \\
& u=-\frac{\gamma x^{2}}{2}+C_{1} x+C_{2} \\
& \text { at } \mathbf{x}=0 \quad \frac{\mathrm{du}}{\mathrm{d} \mathbf{x}}-\phi \mathrm{u}_{0}=0 \quad \text { at } \mathbf{x}=\mathrm{L} \quad \frac{\mathrm{du}}{\mathrm{d} \mathbf{x}}+\phi \mathrm{u}_{\mathrm{L}}=0 \\
& C_{1}=\phi u_{0}=\phi u_{L}-\gamma L \\
& \text { at } \mathrm{x}=0 \quad \mathrm{u}_{0}=\mathrm{C}_{2}=\frac{\mathrm{C}_{1}}{\phi} \quad \mathrm{C}_{1}=\phi \mathrm{C}_{2} \\
& \text { at } x=L \quad u_{L}=\frac{1}{\phi}\left(\gamma L+C_{1}\right)=-\frac{\gamma L^{2}}{2}+C_{1} L+C_{2} \\
& C_{1}=\frac{\gamma L\left(\frac{1}{\phi}-\frac{L}{2}\right)}{\left(\frac{2}{\phi}+L\right)}
\end{aligned}
$$

The heat source is frequently expressed in the form $q=q_{1} e^{\epsilon x}+q_{2}$ and the heat transferred from each surface of the plate is not equal. Substituting the heat function $q$ into the general equation for the steady state.

$$
\begin{aligned}
& \frac{\mathrm{d}^{2} \mathrm{~T}}{\mathrm{dx^{2 }}}+\frac{\mathrm{q}_{1}}{\mathrm{~K}} \mathrm{e}^{\epsilon \mathrm{x}}+\frac{\mathrm{q}_{2}}{\mathrm{~K}}=0 \\
& \text { Let } \mathrm{u}=\mathrm{T}-\mathrm{T}_{\mathrm{c}} \quad \gamma=\frac{\mathrm{q}}{\mathrm{K}} \quad \phi=\frac{\mathrm{h}_{\mathrm{c}}}{\mathrm{K}} \\
& \frac{d^{2} u}{d x^{2}}+\gamma_{1} e^{\epsilon x}+\gamma_{2}=0 \\
& \frac{\mathrm{du}}{\mathrm{dx}}=-\frac{\gamma_{1}}{\epsilon} \mathrm{e}^{\epsilon \mathbf{x}}-\gamma_{2} \mathbf{x}+\mathrm{C}_{1} \\
& u=-\frac{\gamma_{1}}{\epsilon^{2}} e^{e x}-\frac{\gamma_{2} x^{2}}{2}+C_{1} x+C_{2}
\end{aligned}
$$


12

$$
\begin{aligned}
& \text { at } \mathbf{x}=0 \quad \frac{\mathrm{du}}{\mathrm{dx}}-\phi \mathrm{u}_{0}=0 \quad \mathrm{C}_{1}=\phi \mathrm{u}_{0}+\frac{\gamma_{1}}{\epsilon} \\
& \text { at } x=L \quad \frac{d u}{d x}+\phi u_{L}=0 \quad c_{1}=-\phi u_{L}+\frac{\gamma_{1}}{\epsilon} e^{\epsilon L}+\gamma_{2} L \\
& \text { at } x=0 \\
& u_{0}=-\frac{\gamma_{1}}{\epsilon^{2}}+c_{2}=\frac{1}{\phi}\left(c_{1}-\frac{\gamma_{1}}{\epsilon}\right) \\
& \frac{\mathrm{C}_{1}}{\phi}-\mathrm{C}_{2}=\frac{\gamma_{1}}{\epsilon}\left(\frac{1}{\phi}-\frac{1}{\epsilon}\right) \\
& \text { at } x=L \\
& u_{L}=\frac{1}{\phi}\left(-C_{1}+\frac{\gamma_{1}}{\epsilon} e^{\epsilon L}+\gamma_{2} L\right)=-\frac{\gamma_{1}}{\epsilon^{2}} e^{\epsilon L} \\
& -\frac{\gamma_{2} L^{2}}{2}+C_{1} L+C_{2} \\
& C_{1}\left(\frac{1}{\phi}+L\right)+C_{2}=\frac{\gamma_{1}}{\epsilon^{2}} e^{\epsilon L}+\frac{\gamma_{2} L^{2}}{2}+\frac{\gamma_{1} e^{\epsilon L}}{\phi \epsilon}+\frac{\gamma_{2} L}{\phi} \\
& C_{1}=\frac{\left[\frac{\gamma_{1}}{\epsilon}\left(\frac{1}{\phi}-\frac{1}{\epsilon}\right)+\frac{\gamma_{1} e^{\epsilon L}}{\epsilon}\left(\frac{1}{\phi}+\frac{1}{\epsilon}\right)+\gamma_{2} L\left(\frac{1}{\phi}+\frac{L}{2}\right)\right]}{\left(\frac{2}{\phi}+L\right)}
\end{aligned}
$$

The equation of stress is:

$$
\begin{aligned}
& \sigma_{z}=\frac{\alpha \theta E}{(1-\nu)}+\frac{1}{L(1-\nu)} \int_{-L / 2}^{+L / 2} a \theta E d x+\frac{12 x}{L^{3}(1-\nu)} \int_{-L / 2}^{+L / 2} a \theta E x d x \\
& \therefore \sigma_{z}=\frac{\alpha E}{1-\nu}\left\{\theta+\frac{1}{L} \int_{-L / 2}^{+L / 2} \theta d x+\frac{12 x}{L^{3}} \int_{-L / 2}^{+L / 2} \theta x d x\right.
\end{aligned}
$$

0654012 
For Case I

$$
\sigma_{z}=\frac{\alpha E q}{2 K(1-\nu)} \quad\left\{-x^{2}+2 L x-\frac{L^{2}}{12}\right\}
$$

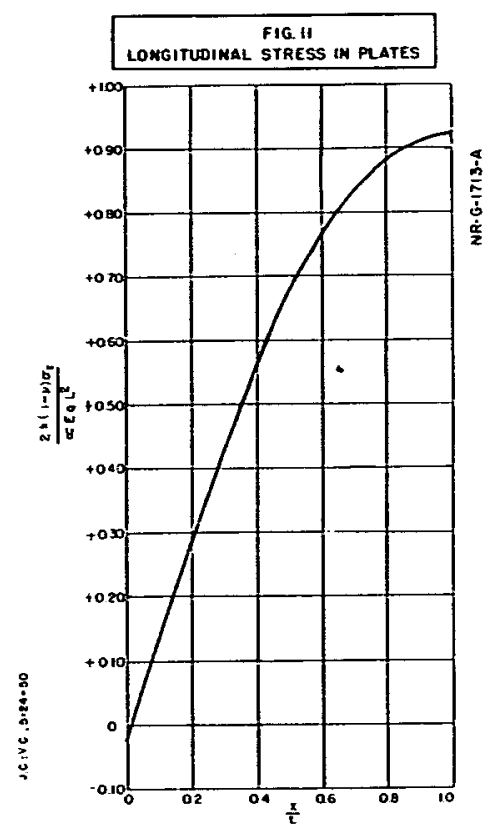

